\title{
Liquid-Liquid Mixing in Stirred Vessels: A Review
}

\author{
REZA AFSHAR GHOTLI,1 ABDUL A. A. RAMAN, 1 \\ SHALIZA IBRAHIM,2 AND SAEID BAROUTIAN 3 \\ 1Department of Chemical Engineering, Faculty of Engineering, \\ University of Malaya, Kuala Lumpur, Malaysia \\ 2Department of Civil Engineering, Faculty of Engineering, \\ University of Malaya, Kuala Lumpur, Malaysia \\ ${ }_{3} S C I O N$, Rotorua, New Zealand
}

Introduction

Mixing is a key and common process to improve homogeneity and uniformity of systems.

Mixing occurs when materials are moved from one area to another in a vessel

(Chen et al., 2005; Rushton, 1956). Nonuniformity of systems can be explained as a

gradient of properties such as concentration, viscosity, temperature, color, concentration,

phase, and temperature (Paul et al., 2004). Mixing operations can be divided

into three main categories, gas-liquid, solid-liquid, and liquid-liquid mixing, the latter

of which is the main focus of the present review. Liquid-liquid mixing plays an important

role in producing and increasing essential interfacial area to improve mass and

heat transfer between phases (O'Rourke and MacLoughlin, 2005; Paul et al., 2004)।

Liquid-liquid mixing is divided into miscible and immiscible liquid-liquid mixing

(van de Vusse, 1955). The term "blending" is used to describe miscible liquid mixing,

while the term "mixing" is used for dispersions of immiscible liquids or the formation

of emulsions (Jakobsen, 2008; Rushton, 1956). The dispersion of immiscible

liquids is used to mix water and hydrocarbons and acidic or alkaline solutions

combined with organic liquids (Coker, 2001) and produce various types of emulsion products (Jakobsen, 2008). Liquid-liquid mixing is applicable for special process

objectives such as solvent extraction and removal or addition of heat (Paul et al., 2004). The fluid dynamic characteristics of liquid-liquid mixing produces several phenomena such as drop breakup and coalescence (Wichterle, 1995; Wang and 
Calabrese, 1986; Sathyagal et al., 1996), mean flow pattern and turbulence (Ibrahim and Nienow, 1995; Norwood and Metzner, 1960), drop suspension, interfacial area, and drop size distribution (DSD) (Podgorska and Baldyga, 2001; Fernandes and Sharma, 1967), possible phase inversion (Norato et al., 1998), and the influence of system composition as well as of small amounts of impurities (Laurenzi et al., 2009). These phenomena are complicated and cause liquid-liquid dispersion to be one of the most difficult processes in a number of industries. On the other hand, blending of miscible liquid is a very simple operation that is achieved by two mutually soluble liquids in the absence of resistivity to dissolution at the fluid interface (Paul et al., 2004). Generally, blending of miscible liquids happens slowly by molecular diffusion and natural convection. Thus, agitation systems can apply forced convection to obtain homogeneity more rapidly (Rushton, 1956).

Tanks and vessels are the most accessible and universal equipment used in a wide range of process industries such as esterification and hydrolysis (Paul et al., 2004). Because natural diffusion in liquids is slow, agitators that provide high shear and good pumping capacity are common choices for liquid-liquid dispersion and emulsification because they improve diffusivity. Nevertheless, it is possible to waste large amounts of input energy through inappropriate system selection (Holland and Bragg, 1995). Furthermore, inadequate understanding of mixing could result in undesirable product quality and increased production costs. Mixing operations are often complex. They not only require understanding the fluid flow aspects, but also consideration of the mechanical equipment and power requirements (Chen et al., 2005).

\section{Parameters That Define Liquid-Liquid Mixing Performance Mixing Time}

Mixing time is one of the most significant parameters in liquid-liquid mixing and scale-up because it is also the time required to obtain a defined degree of uniformity (Montante et al., 2005; Jakobsen, 2008). It is the time required to achieve desirable mixing and homogeneity throughout the tank. Impeller speed, the diameter of the vessels and impellers, the number and placement of baffles, and fluid characteristics such as viscosity are the effective parameters for determining mixing time (Jakobsen, 2008; Doran, 1995).

Several mixing time correlations have already been developed to estimate and compute the mixing time in different standard-baffled mixing vessels and with various types of impellers such as Rushton turbines, pitched blades, and propellers 
(Coker, 2001). There are, however, some limitations to the wide application of these correlations. Most of these equations are based on experiments with standard tank geometries and a single impeller. Consequently, they are not useful for multiple-impeller systems. Furthermore, comparison of the different research results is not a simple task because such factors as measurement and experimental methods, tank and impeller geometry, location of tracer injection, and detection method vary widely from study to study (Jahoda et al., 2007). Nere et al. (2003) did an expanded review of mixing time correlations and divided them into five categories: (i) models based on experimental data with various design and operating parameters; drawbacks of these models are due to limited application and not being reliable for other systems, especially in scale-up; (ii) models based on bulk flow, which assumes that the process is controlled by the bulk or convective flow; (iii) models based on dispersion; (iv) models that segregate the whole stirred vessel into a network of interconnected zones; and (v) CFD models. Drawbacks and effectiveness of these models are extensively discussed by Nere et al. (2003).

Several methods such as the decolorization reaction of iodine and sodium thiosulfate in water (Nomura et al., 1997; Yao et al., 1998; Hiraoka et al., 2001; Kato et al., 2005), electrical conductivity probes (Kramers et al., 1953; Biggs, 1963; Wesselingh, 1975; Kumaresan et al., 2005; Woziwodzki, 2011), temperature pulse (Mayr et al., 1992: Karcz et al., 2005; Szoplik and Karcz, 2005; Slemenik Per_se

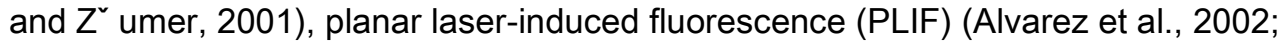
Crimaldi, 2008; Zadghaffari et al., 2009), particle image velocimetry (PIV) (Zalc et al., 2001; Alvarez et al., 2005), acid-base neutralization reaction (Rice et al., 1964; Norwood and Metzner, 1960; Lamberto et al., 1996; Szalai et al., 2004; Woziwodzki and Je_drzejczak, 2011), and electrical resistance tomography (ERT) (Pakzad et al., 2008) have been used for mixing time determination. Mixing time may vary for each technique as a result of the variety of homogeneity degree measurements (Coker, 2001). Commonly, these methods are based on visual observation. A small amount of tracer is added to the bulk fluid and is monitored. Various types of tracers, principally hot water (Hoogendoorn and den Hartog, 1967; Mayr et al., 1992; Karcz et al., 2005), sodium chloride solution (Kumaresan et al., 2005; Woziwodzki et al., 2010), or fluorescent dye (rhodamine) (Alvarez et al., 2002, 2005; Szalai et al., 2004; Hu et al., 2010), have been employed for determination of mixing time. The radioactive tracer technique was suggested as a good alternative by Nere et al. (2003) due to its nonintrusiveness and ability to be used with nontransparent vessels. Very frequently, results differ considerably because of 
the number and location of probes used to take samples as well as tracer injection time and location (Lamberto et al., 1999).

In addition, various parameters, such as impeller design, impeller diameter, tank diameter, impeller clearance, impeller eccentricity, baffles, and presence of a draft tube can affect mixing time. Van de Vusse et al. (1955) demonstrated that at low stirrer speeds baffling has little effect on mixing time. Kumaresan et al. (2005) observed that an increase in the number of baffles causes a slight reduction in mixing time. They also presented evidence of a considerable reduction in mixing time, even up to $60 \%$, for a hydrofoil impeller surrounded by a long draft tube compared to a pitched-blade impeller. Ochieng et al. (2008) also reported 50\% reduction in mixing time using a draft tube with a single Rushton turbine with only a small clearance between the impeller and the tank bottom. They used two conductivity and decolorization methods. Their results showed $35 \%$ reduction in mixing time at low impeller clearance in comparison with the standard configuration. The comparison of mixing time for pitched-blade turbines (down flow (PTD) and up flow (PTU)) with that for a disk turbine by Rewatkar and Joshi (1991) indicated that the pitched-blade turbines are more energy efficient than the disk turbine. Better performance of the pitched-blade turbines was explained on the basis of the flow pattern produced by these impellers. Increasing the impeller diameter causes a reduction in mixing time values. Increasing the impeller diameter results in larger average circulation velocity, and thus causes a decrease in the mixing time value. Patwardhan and Joshi (1999) also determined the mixing time for around 40 axial-flow impellers. The impellers were varied in angle, twist, width, diameter, location, and pumping direction. The changes in blade angle showed that use of impellers with a blade angle of 50_resulted in the shortest mixing time. Mixing time decreased with each incremental increase in impeller blade width. They also observed that an increase in the ratio of impeller diameter $(D)$ to tank diameter $(T)$ up to $D=T 1 / 41=2$ for a pitched-blade turbine (PBTD 45) impeller at impeller clearance $(C)^{1 / 4} \mathrm{~T}=3$ caused the mixing time to decrease. However, if the $D=T$ ratio is increased beyond $D=T 1 / 41=2$, mixing time tends to begin increasing. Further results showed that mixing time increased with clearance reduction. Determination of mixing time by Zhao et al. (2011) in a kerosene and water system indicated an increase in mixing time with a pitched-blade turbine PTU impeller. The PTU transfers the flow upward, and as kerosene is lighter than water it thus tends to coalesce and stay on the surface, resulting in poor dispersion of the oil and increasing mixing time. Furthermore, the experimental data indicated a reduction in mixing time by decreasing the impeller clearance in the range of 
$\mathrm{T}=3$ to $\mathrm{T}=6$. Investigation of mixing time for miscible liquids in batch operation by van de Vusse et al. (1955) showed that in the turbulent flow region, mixing time is directly related to impeller pumping capacity.

Impeller clearance reduction and use of a draft tube decrease mixing time and result in better mixing (Ochieng and Onyango, 2008). The axial mixing improves at low clearance because of interaction between the flow stream and the bottom wall, which produces a one-loop flow pattern (Montante et al., 2001). Enhancement of axial velocity and the one-loop flow pattern causes the transfer rate of the tracer through the tank to intensify from the bottom to the top area. Subsequently, reduction in mixing time results in better mixing (Ochieng and Onyango, 2008). The review showed that mixing time is strongly affected by any changes in geometrical ratio such as $\mathrm{D}=\mathrm{T}$ and $\mathrm{C}=\mathrm{T}$ and impeller design.

\section{Circulation Time}

Evaluation of the mean bulk fluid motion produced by the pumping of liquid with impellers in mixing vessels is done by circulation time. Determination of circulation and mixing times are helpful in recognizing the scalar transport in a tank. The mean circulation time describes an average value for all the fluid components in the vessel and also indicates how fast the bulk fluids are transported through a tank. On the other hand, mixing time determines the time at which the whole of the tank is perfectly mixed or when the system reaches uniformity. Generally, the mean circulation time is significantly shorter than mixing time in a tank (Jakobsen, 2008). For example, mixing time for a single-phase liquid in a stirred vessel is approximately four times the circulation time (Doran, 1995; Nienow, 1997). The evaluation of mixing time and circulation time performed by Partwardhan and Joshi (1999) also revealed that the mixing time for a 45_-pitch down-flow blade impeller (PBTD45) is slightly more than three times the circulation time. Circulation time is related to the total volumetric flow rate and entrainment flow of the tank and the pumping capacity of the impeller, and is defined by the following assumption (Jakobsen, 2008):

Full text is available at :

www.tandfonline.com/doi/abs/10.1080/00986445.2012.717313 